\title{
An Extension of One-Period Nash Equilibrium Model in Non-Life Insurance Markets
}

\author{
G. Battulga1, L. Altangerel2*, G. Battur ${ }^{1}$ \\ ${ }^{1}$ Department of Applied Mathematics, National University of Mongolia, Ulaanbaatar, Mongolia \\ ${ }^{2}$ Faculty of Mathematics, Computer and Natural Sciences, German-Mongolian Institute for Resources and Technology, Nalaikh, \\ Mongolia \\ Email: battulga.g@seas.num.edu.mn, *altangerel@gmit.edu.mn, battur@seas.num.edu.mn
}

How to cite this paper: Battulga, G., Altangerel, L. and Battur, G. (2018) An Extension of One-Period Nash Equilibrium Model in Non-Life Insurance Markets. Applied Mathematics, 9, 1339-1350. https://doi.org/10.4236/am.2018.912087

Received: November 11, 2018

Accepted: December 17, 2018

Published: December 20, 2018

Copyright (c) 2018 by authors and Scientific Research Publishing Inc. This work is licensed under the Creative Commons Attribution International License (CC BY 4.0).

http://creativecommons.org/licenses/by/4.0/

\begin{abstract}
This paper deals with an extension of the one-period model in non-life insurance markets (cf. [1]) by using a transition probability matrix depending on some economic factors. We introduce a multi-period model and in each period the solvency constraints will be updated. Moreover, the model has the inactive state including some uninsured population. Similar results on the existence of premium equilibrium and sensitivity analysis for this model are presented and illustrated by numerical results.
\end{abstract}

\section{Keywords}

Nash Equilibrium Model, Variational Inequalities, Transition Matrix, Non-Life Insurance Markets

\section{Introduction}

We consider $I$ insurers competing in a market of $n$ policyholders or insureds. Assume that the policyholders can decide either to renew the policy with the present insurer or switch to one of the competitors.

According to Dutang et al. in [1], there are two non-cooperative game theory models in insurance markets: the Bertrand oligopoly, where insurers set premiums and Cournot oligopoly, where insurers choose optimal values of insurance coverage. Some extensions of these models have been investigated by various authors (see [1] and references therein). The game theoretic approach has received a great deal of attention by many authors, who contributed in various ways (see [2] [3] [4] and references therein).

By considering a lapse and an aggregate loss models for policyholders, the Bertrand model of Rees et al. (cf. [5]) has been extended in [1]. They showed the 
suitability of non-cooperative game theory for insurance market modelling. Moreover, they introduced the solvency constraints first time. As usual, "game" for insurers means to set premium for which policies are offered to the policyholders.

It would be interesting to investigate a model from the perspective of insureds' behavior how they can react on current economic situation. If the economy is getting better, then insureds including individuals and companies are interested in having insurance contracts, contrariwise they might be uninsured. On the other hand, the success and achievements of insurers in the coverage period can attract customers to keep insurance contracts. Otherwise, they have a risk to lose customers. Therefore, attracting insureds could depend on economic factors such as macroeconomic variables and financial data of insurance companies.

This paper aims to extend the one-period model in non-life insurance markets (see [1]) by using a transition probability matrix depending on some economic factors. We consider a model with a multi-period and assume that the solvency constraints will be updated in each period. Moreover, our model has the inactive state which means some insureds are uninsured.

The rest of the paper is structured as follows. In Section 2, we give a short summary of the one-period model. Section 3 deals with an extension of the one-period model and some assertions related to the existence of premium equilibrium and sensitivity analysis are presented. In conclusion, in Section 4, some numerical results are given.

\section{The One-Period Model}

In this section, we provide a short overview of the one-period model investigated in [1]. Let $\left(x_{1}, \cdots, x_{I}\right) \in \mathbb{R}^{I}$ be a price vector, where $x_{j}$ represents the premium of insurer $j$. Once the premium is set by all insurers, the insureds choose to renew or to lapse from their current insurer. Then, insurers pay claims, according to their portfolio size, during the coverage year.

Let $Y_{i}$ be the aggregate loss of policy $i$ during the coverage period. We assume that $Y_{i}, i \in\{1, \cdots, n\}$ are independent and identically distributed (i.i.d.) random variables. The aggregate claim amount is $S_{j}(x)=\sum_{i=1}^{N_{j}(x)} Y_{i}$, where $N_{j}(x)$ is the portfolio size of insurer $j$ given the price vector $x$.

Let $n_{j}$ be the initial portfolio size of insurer $j$, i.e., $\sum_{j=1}^{I} n_{j}=n$. We assume that insurer $j$ maximizes the expected profit of renewing policies defined as

$$
O_{j}(x)=\frac{n_{j}}{n}\left(1-\beta_{j}\left(\frac{x_{j}}{m_{j}(x)}-1\right)\right)\left(x_{j}-\pi_{j}\right),
$$

where $\pi_{j}$ is the break-even premium $j$ expressed as

$$
\pi_{j}=w_{j} \bar{a}_{j, 0}+\left(1-w_{j}\right) \bar{m}_{0}
$$

and $m_{j}(x)$ is a market premium proxy which is the mean price of the other 
competitors

$$
m_{j}(x)=\frac{1}{I-1} \sum_{k \neq j} x_{k} .
$$

By $\bar{a}_{j, 0}$ and $\bar{m}_{0}$, we denote the actuarial premium based on the past loss experience of insurer $j$ and the market premium, respectively. $w_{j} \in[0,1]$ is the credibility factor of insurer $j$ and $\beta_{j}>0$ is the elasticity parameter.

In addition to maximizing a certain objective function, insurers must satisfy a solvency constraint imposed by the regulator. A simplification is to approximate a $q$-quantile $Q(n, q)$ of aggregate claim amount of $n$ i.i.d. risks by a bilinear function of $n$ and $\sqrt{n}$

$$
Q(n, q)=E(Y) n+k_{q} \sigma(Y) \sqrt{n}
$$

where the solvency coefficient $k_{q}$ has to be determined and $Y$ is the generic individual claim severity variable. $E(\cdot)$ and $\sigma(\cdot)$ are a mean and standard deviation of a randon variable. Using the approximation the solvency capital requirement SCR is deduced as

$$
S C R_{q} \approx k_{q} \sigma(Y) \sqrt{n} .
$$

Then the solvency constraint function can be defined as follows

$$
g_{j}\left(x_{j}\right)=\frac{K_{j}+n_{j}\left(x_{j}-\pi_{j}\right)\left(1-e_{j}\right)}{k_{q} \sigma(Y) \sqrt{n}} \geq 1,
$$

where $e_{j}$ is the expense rate as a percentage of gross written premium.

The one-period Nash equilibrium model in non-life insurance markets becomes

$$
\max _{x_{j} \in X_{j}} O_{j}(x), j=1, \cdots, I
$$

where

$$
\begin{aligned}
X_{j} & :=\left\{x_{j} \in[\underline{x}, \bar{x}] \mid g_{j}\left(x_{j}\right) \geq 0\right\} \\
& =\left\{x_{j} \in[\underline{x}, \bar{x}] \mid K_{j}+n_{j}\left(x_{j}-\pi_{j}\right)\left(1-e_{j}\right) \geq k_{q} \sigma(Y) \sqrt{n}\right\},
\end{aligned}
$$

and $\underline{x}, \bar{x}$ are the minimum and the maximum premium, respectively.

\section{Extension of the One-Period Model}

This section deals with an extension of the one-period model considered from the perspective economic factors. Let $m$ be number of periods. To consider a possible extension of the model with $m$-period, we assume that policyholders will react on the current economic situation i.e., if the economy is getting better, then they have interests to be insured. As before, we assume that the market has $I$ insurers and $n$ insureds. Let $z(k) \in \mathbb{R}^{q}$ be economic factor in $k$ th period and $\gamma_{i j}(k) \in \mathbb{R}^{q}$ be a vector of economic weights in $k$ th period with respect to the movement from insurer $i$ to $j$. We introduce a transition matrix (see [6]) 
describing insureds' movement to insurers.

$$
P(k)=\left(\begin{array}{ccccc}
p_{1,1}(k) & p_{1,2}(k) & \cdots & p_{1, I}(k) & p_{1, I+1}(k) \\
p_{2,1}(k) & p_{2,2}(k) & \cdots & p_{2, I}(k) & p_{2, I+1}(k) \\
\vdots & \vdots & \ddots & \vdots & \vdots \\
p_{I, 1}(k) & p_{I, 2}(k) & \cdots & p_{I, I}(k) & p_{I, I+1}(k) \\
p_{I+1,1}(k) & p_{I+1,2}(k) & \cdots & p_{I+1, I}(k) & p_{I+1, I+1}(k)
\end{array}\right),
$$

where $p_{i, j}(k)$ denotes the probability for customers to switch from insurer $i$ to $j$ in $k$ th period. $(I+1)$ th column corresponds to uninsured ones whose state can be called inactive. According to [7] (see, also [1]), the transition probability can be modelled as

$$
p_{i, j}(k)= \begin{cases}\frac{1}{1+\sum_{l=1}^{I} \exp \left(\left\langle\gamma_{i l}(k), z(k)\right\rangle\right)} & \text { if } j=I+1 \\ \frac{\exp \left(\left\langle\gamma_{i j}(k), z(k)\right\rangle\right)}{1+\sum_{l=1}^{I} \exp \left(\left\langle\gamma_{i l}(k), z(k)\right\rangle\right)} & \text { if } j \neq I+1,\end{cases}
$$

where $\langle\cdot, \cdot\rangle$ is the Euclidean inner product. If the economy is deteriorate in $k$ th period, some insureds don't want to keep insurance contracts, therefore $p_{i, j}(k), j=1, \cdots, I$ will decrease and $p_{i, I+1}$ will increase. In $k$ th period, the portfolio size $N_{j}(k)$ of insurer $j$ for the next period is determined by the sum of renewed policies and businesses coming from other insurers. Hence

$$
\begin{aligned}
N_{j}(k) & =N_{j}(k-1) p_{j, j}(k)+\sum_{i=1, i \neq j}^{I+1} N_{i}(k-1) p_{i, j}(k) \\
& =\sum_{i=1}^{I+1} N_{i}(k-1) p_{i, j}(k),
\end{aligned}
$$

where $N_{j}(0)=n_{j}$. Let $r_{f}$ be a risk free rate and $v=\frac{1}{1+r_{f}}$ be a discount factor. Based on [1], the insurer $j$ maximizes the present value of expected profit of renewing policies defined as

$$
O_{m}^{j}(x)=\sum_{k=1}^{m} \frac{v^{k} N_{j}(k)}{n}\left(1-\beta_{j}(k)\left(\frac{x_{j}^{k}}{m_{j}\left(x^{k}\right)}-1\right)\right)\left(x_{j}^{k}-\pi_{j}(k)\right),
$$

where $x=\left(x_{1}^{\mathrm{T}}, \cdots, x_{I}^{\mathrm{T}}\right)^{\mathrm{T}}, \quad x_{j}=\left(x_{j}^{1}, \cdots, x_{j}^{m}\right)^{\mathrm{T}}$ for $j=1, \cdots, I$, and $m_{j}\left(x^{k}\right)=\frac{1}{I-1} \sum_{i \neq j} x_{i}^{k}$. The solvency constraints for insurer $j$ can be redefined as

$$
g_{\ell}^{j}\left(x_{j}\right)=\frac{K_{j}+\sum_{k=1}^{\ell} v^{k} N_{j}(k)\left(x_{j}^{k}-\pi_{j}(k)\right)\left(1-e_{j}(k)\right)}{k_{q} \sigma(Y) \sqrt{\sum_{k=1}^{\ell} v^{k} N_{j}(k)}}-1, \text { for } \ell=1,2, \cdots, m
$$

Then, the strategy set of each player $j, j \in\{1, \cdots, I\}$ is 


$$
\begin{aligned}
X_{m}^{j}:= & \left\{x_{j} \in[\underline{x}, \bar{x}]^{m} \mid g_{\ell}^{j}\left(x_{j}\right) \geq 0, \ell=1, \cdots, m\right\} \\
= & \left\{x_{j} \in[\underline{x}, \bar{x}]^{m} \mid K_{j}+\sum_{k=1}^{\ell} v^{k} N_{j}(k)\left(x_{j}^{k}-\pi_{j}(k)\right)\left(1-e_{j}(k)\right)\right. \\
& \left.\geq k_{q} \sigma(Y) \sqrt{\sum_{k=1}^{\ell} v^{k} N_{j}(k)}, \ell=\overline{1, m}\right\}
\end{aligned}
$$

Now we give some similar results for $m$-period model.

Proposition 1. The m-period insurance game with I players whose objective functions and solvency constraints are defined by (1) and (2), respectively, admits a unique Nash premium equilibrium.

Proof: In a similar way as in [1] and by Theorem 1 in [8], we can verify the existence of a Nash equilibrium. On the other hand, since for any $\left(x_{j}^{1}, \cdots, x_{j}^{\ell-1}, x_{j}^{\ell+1}, \cdots, x_{j}^{m} ; x_{-j}\right)$, the function $O_{m}^{j}(x)$ is strictly concave and differentiable with respect to $x_{j}^{\ell}$, for $\forall x, y \in \mathbb{R}^{I m}$ it hold

$$
\left\langle\nabla_{x_{j}} O_{m}^{j}(x), y_{j}-x_{j}\right\rangle>O_{m}^{j}(y)-O_{m}^{j}(x),
$$

and

$$
\left\langle\nabla_{x_{j}} O_{m}^{j}(y), x_{j}-y_{j}\right\rangle>O_{m}^{j}(x)-O_{m}^{j}(y) .
$$

Adding both inequalities, we have

$$
\begin{aligned}
& \left\langle\nabla_{x_{j}} O_{m}^{j}(x), y_{j}-x_{j}\right\rangle+\left\langle\nabla_{x_{j}} O_{m}^{j}(y), x_{j}-y_{j}\right\rangle \\
& >O_{m}^{j}(y)-O_{m}^{j}(x)+O_{m}^{j}(x)-O_{m}^{j}(y)=0 .
\end{aligned}
$$

Denoting by $r=(1, \cdots, 1)^{\mathrm{T}} \in \mathbb{R}^{I}$ and taking the sum by $j, j=1, \cdots, I$, we obtain that

$$
\sum_{j=1}^{I} r_{j}\left\langle\nabla_{x_{j}} O_{m}^{j}(x), y_{j}-x_{j}\right\rangle+\sum_{j=1}^{I} r_{j}\left\langle\nabla_{x_{j}} O_{m}^{j}(y), x_{j}-y_{j}\right\rangle>0, \forall x, y \in \mathbb{R}^{m I}
$$

which guarantees the uniqueness of the equilibrium (cf. Theorem 2 in [8]).

Proposition 2. Let $x^{*}$ be the premium equilibrium of the m-period insurance game with I players.

1) If all solvency constraints are either active or inactive, then for each player $j$ and period $k$, the corresponding equilibrium $\left.x_{j}^{k^{*}} \in\right] \underline{x}, \bar{x}[$ depends on the parameters in the following way:

a) It increases with break-even premiums $\pi_{j}(k)$, solvency coefficient $k_{q}$, loss volatility $\sigma(Y)$, expense rate $e_{j}(k)$, and risk free rate $r_{f}$ for $k \geq 2$ and

b) Decreases with sensitivity parameter $\beta_{j}(k)$, capital $K_{j}$ for $k=1$, and, portfolio size $N_{j}(\ell), \ell=1, \cdots, k$ for $k \geq 2$.

2) If all constraint functions are inactive, then the premium equilibrium is a solution of the linear system of equations

$$
M_{\beta} x^{*}=v,
$$

where 


$$
\begin{gathered}
M_{\beta}=\left(\begin{array}{ccccc}
A_{1} & 0 & 0 & \cdots & 0 \\
0 & A_{2} & 0 & \cdots & 0 \\
\vdots & \vdots & \vdots & \ddots & \vdots \\
0 & 0 & 0 & \cdots & A_{m}
\end{array}\right), \\
v=\left(\beta_{1}(1) \pi_{1}(1), \cdots, \beta_{I}(1) \pi_{I}(1), \cdots, \beta_{1}(m) \pi_{1}(m), \cdots, \beta_{I}(m) \pi_{I}(m)\right)^{\mathrm{T}},
\end{gathered}
$$

and

$$
A_{k}=\left(\begin{array}{cccc}
2 \beta_{1}(k) & -\frac{1+\beta_{1}(k)}{I-1} & \cdots & -\frac{1+\beta_{1}(k)}{I-1} \\
-\frac{1+\beta_{2}(k)}{I-1} & 2 \beta_{2}(k) & \cdots & -\frac{1+\beta_{2}(k)}{I-1} \\
\vdots & \vdots & \ddots & \vdots \\
-\frac{1+\beta_{I}(k)}{I-1} & -\frac{1+\beta_{I}(k)}{I-1} & \cdots & 2 \beta_{I}(k)
\end{array}\right), k=1, \cdots, m .
$$

Proof: The KKT conditions for the premium equilibrium $x_{j}^{*}$ of insurer $j$ has the following form:

$$
\left\{\begin{array}{l}
\nabla_{x_{j}} O_{j}\left(x^{*}\right)+\sum_{\ell=1}^{m} \lambda_{i \ell}^{j^{*}} \nabla_{x_{j}} g_{\ell}^{j}\left(x_{j}^{*}\right)+\lambda_{2}^{j^{*}}-\lambda_{3}^{j^{*}}=0, \\
\lambda^{j^{*}}=\left(\lambda_{1}^{j^{*}}, \lambda_{2}^{j^{*}}, \lambda_{3}^{j^{*}}\right)^{\mathrm{T}} \geq 0, \lambda_{1}^{j^{*}} \in \mathbb{R}^{m}, \lambda_{2}^{j^{*}} \in \mathbb{R}^{m}, \lambda_{3}^{j^{*}} \in \mathbb{R}^{m}, \\
g_{k}^{j}\left(x_{j}^{*}\right) \geq 0, x_{j}^{k^{*}}-\underline{x} \geq 0, \bar{x}-x_{j}^{k} \geq 0, k=1, \cdots, m, \\
\lambda_{1 k}^{j^{*}} g_{k}^{j}\left(x_{j}^{*}\right)=0, \lambda_{2 k}^{j^{*}}\left(x_{j}^{k^{*}}-\underline{x}\right)=0, \lambda_{3 k}^{j^{*}}\left(\bar{x}-x_{j}^{k}\right)=0, k=1, \cdots, m .
\end{array}\right.
$$

$k$-th component from the first equation of the system becomes

$$
\frac{\partial}{\partial x_{j}^{k}} O_{m}^{j}\left(x^{*}\right)+\sum_{\ell=1}^{m} \lambda_{i \ell}^{j^{*}} \frac{\partial}{\partial x_{j}^{k}} g_{\ell}^{j}\left(x_{j}^{*}\right)+\lambda_{2 k}^{j^{*}}-\lambda_{3 k}^{j^{*}}=0 .
$$

1) Let $\left.x_{j}^{k^{*}} \in\right] \underline{x}, \bar{x}\left[\right.$. Then $\lambda_{2 k}^{j^{*}}=\lambda_{3 k}^{j^{*}}=0$. We consider two cases.

a) Let us assume that the solvency constraints are all inactive, i.e., $g_{\ell}^{j}\left(x_{j}^{*}\right)>0, \ell=1, \cdots, m$. Then, insurer $j$ s premium equilibrium verifies

$$
\begin{aligned}
\frac{\partial}{\partial x_{j}^{k}} O_{m}^{j}\left(x^{*}\right) & =0, \text { i.e. } \\
& \frac{v^{k} N_{j}(k)}{n}\left(1-2 \beta_{j}(k) \frac{x_{j}^{k^{*}}}{m_{j}\left(x^{k^{*}}\right)}+\beta_{j}(k)+\beta_{j}(k) \frac{\pi_{j}(k)}{m_{j}\left(x^{k^{*}}\right)}\right)=0 .
\end{aligned}
$$

Let $x_{j}^{k}(y):=\left(x_{j}^{1}, \cdots, x_{j}^{k-1}, y, x_{j}^{k+1}, \cdots, x_{j}^{m} ; x_{-j}\right)^{\mathrm{T}}$. In order to investigate the sensitivity depending on parameter $z$, let us define the function $F_{x}^{k, j}$ as

$$
F_{x}^{k, j}(z, y):=\frac{\partial}{\partial x_{j}^{k}} O_{m}^{j}\left(x_{j}^{k}(y), z\right),
$$

and consider the equation of the form $F_{x}^{k, j}(z, y)=0$. Under assumptions that partial derivatives of $F_{x}^{k, j}$ exist and are continuous at $\left(z_{0}, y_{0}\right)$, and also $\frac{\partial F_{x}^{k, j}}{\partial y}\left(z_{0}, y_{0}\right) \neq 0$, by the implicit function theorem, there exists a function $\varphi$ 
defined in a neighbourhood of $\left(z_{0}, y_{0}\right)$ such that $F_{x}^{k, j}(z, \varphi(z))=0$ and $\varphi\left(z_{0}\right)=y_{0}$. The derivative of $\varphi$ is given by

$$
\phi^{\prime}(z)=-\left.\frac{\frac{\partial F_{x}^{k, j}}{\partial z}}{\frac{\partial F_{x}^{k, j}}{\partial y}}\right|_{y=\varphi(z)} .
$$

In our case, we have

$$
\frac{\partial F_{x}^{k, j}}{\partial y}(z, y)=\frac{\partial^{2} O_{m}^{j}}{\partial x_{j}^{k 2}}=-2 \beta_{j}(k) \frac{v^{k} N_{j}(k)}{n \cdot m_{j}\left(x^{k}\right)}<0 .
$$

As a consequence, it holds

$$
\operatorname{sign}\left(\phi^{\prime}(z)\right)=\operatorname{sign}\left(\frac{\partial F_{x}^{k, j}}{\partial z}(z, \phi(z))\right) .
$$

i) Let $z:=\pi_{j}(k)$. Then

$$
\frac{\partial F_{x}^{k, j}}{\partial z}(z, y)=\frac{v^{k} N_{j}(k) \beta_{j}(k)}{n \cdot m_{j}\left(x^{k}\right)}>0 .
$$

In other words, the function $\pi_{j}(k) \rightarrow x_{j}^{k^{*}}\left(\pi_{j}(k)\right)$ is increasing.

ii) Let $z$ be the sensitivity coefficient $\beta_{j}(k)$. Then, we have

$$
\frac{\partial F_{x}^{k, j}}{\partial z}(z, y)=\frac{v^{k} N_{j}(k)}{n}\left(-2 \frac{y}{m_{j}\left(x^{k}\right)}+1+\frac{\pi_{j}(k)}{m_{j}\left(x^{k}\right)}\right) .
$$

By using (4), we obtain that

$$
\frac{\partial F_{x}^{k, j}}{\partial z}(z, \phi(z))=\frac{v^{k} N_{j}(k)}{n} \cdot \frac{-1}{z}<0 .
$$

Therefore, the function $\beta_{j}(k) \rightarrow x_{j}^{k^{*}}\left(\beta_{j}(k)\right)$ is decreasing.

b) If the solvency constraints are all active, then the premium equilibrium satisfies $g_{\ell}^{j}\left(x_{j}^{*}\right)=0$, for $\ell=1, \cdots, m$ and consequently, one get

$$
\begin{gathered}
x_{j}^{1^{*}}=\pi_{j}(1)+\frac{k_{q} \sigma(Y) \sqrt{v N_{j}(1)}-K_{j}}{v N_{j}(1)\left(1-e_{j}(1)\right)}, \\
x_{j}^{\ell^{*}}=\pi_{j}(\ell)+\frac{k_{q} \sigma(Y)}{\left(\sqrt{\sum_{k=1}^{\ell} v^{k} N_{j}(k)}+\sqrt{\sum_{k=1}^{\ell-1} v^{k} N_{j}(k)}\right)\left(1-e_{j}(\ell)\right)}, \ell=2, \cdots, m .
\end{gathered}
$$

From (5), we can verify directly that $x_{j}^{k^{*}}$ is an increasing function of $\pi_{j}(k)$, $k_{q}, e_{j}(k)$ and $r_{f}$ for $k \geq 2$. Moreover, it is a decreasing function of $K_{j}$ for $k=1$ and $N_{j}(\ell), \ell=1, \cdots, k$ for $k \geq 2$.

2) If all constraints are inactive at a Nash equilibrium $x^{*}$, then taking into account $m_{j}\left(x^{k}\right)=\frac{1}{I-1} \sum_{i \neq j} x_{i}^{k}$ and from (4) follows that

$$
2 \beta_{j}(k) x_{j}^{k^{*}}-\left(1+\beta_{j}(k)\right) \frac{1}{I-1} \sum_{i \neq j} x_{i}^{k^{*}}=\beta_{j}(k) \pi_{j}(k), \forall j, k .
$$


This system can be rewritten in matrix form as $M_{\beta} x=v$. As in [1] mentioned, we can see that the matrix $M_{\beta}$ is strictly diagonally dominant if the conditions $\beta_{j}(k)>1, j=\overline{1, I}, k=\overline{1, m}$ are fulfilled. Under this condition $M_{\beta}$ is invertible and therefore $x^{*}=M_{\beta}^{-1} v$.

Remark 1. If $x_{j}^{k^{*}}=\underline{x}$ or $\bar{x}$, then the premium equilibrium is independent of those parameters.

Remark 2. For a game with one leader and $I-1$ followers with payoff functions $O_{m}^{j}$ and the strategy set $X_{m}^{j}$, a Stackelberg equilibrium is the problem that consists in finding a vector $\bar{x}=\left(\bar{x}_{1}^{\mathrm{T}}, \cdots, \bar{x}_{I}^{\mathrm{T}}\right), \bar{x}_{j}=\left(\bar{x}_{j}^{1}, \cdots, \bar{x}_{j}^{m}\right)$ such that $\bar{x}_{1}$ solves the problem

$$
\sup _{y \in X_{m}^{1}} O_{1}\left(y ; x_{2}, \cdots, x_{I}\right),
$$

where $\left(x_{2}, \cdots, x_{I}\right)$ is a Nash equilibrium for the game with the $I-1$ followers and given strategy $x_{1}$ for insurer 1 which is assumed to be a leader. In this case, it is not difficult to show the existence of Stackelberg equilibrium (cf. [1]).

\section{Numerical Experiments}

In this section we show some numerical results dealing with sensitivity analysis presented in Proposition 2 in Section 3. Let us notice that the Nash equilibrium model can be reduced to the variational inequality problem which consists in finding $x \in \Omega:=X_{m}^{1} \times X_{m}^{2} \times \cdots \times X_{m}^{I}$ such that

$$
(V I)\langle F(x), y-x\rangle \geq 0, \forall y \in \Omega
$$

where $F(x)=\left(\nabla_{x_{j}} O_{m}^{j}(x)\right)_{j=1}^{I}$. In order solve the problem $(V I)$, we apply the hyperplane projection algorithm (see [9] and [10]). We consider three player's game and let $m=3$.

1) Base case:

Table 1 shows that if we use the data from [1] in each period, then we get the same results.

2) Scenario 1:

Table 2 shows results for the case if elasticity parameter of first player increases up to 3.5 in three periods.

3) Scenario 2:

Table 3 presents results for the case if elasticity parameters of all players increase in Period 2. Then, premium equilibriums are changed only in Period 2.

4) Scenario 3:

In this case, we assume that break-even premium for player 1 in Period 1 and for player 3 in Period 3 are increasing and break-even premium for player 2 in Period 2 is decreasing. Then, premium equilibriums in Period 1 and Period 3 for players 1 and 3 are increasing, but premium equilibrium in Period 2 for player 2 is decreasing as compared with "Base case" (see Table 4).

5) Scenario 4: 
Table 1. Basic case.

\begin{tabular}{|c|c|c|c|c|c|c|c|c|c|}
\hline \multirow{2}{*}{$\begin{array}{l}\text { Player } \\
\text { Period }\end{array}$} & \multicolumn{3}{|c|}{ I } & \multicolumn{3}{|c|}{ II } & \multicolumn{3}{|c|}{ III } \\
\hline & 1 & 2 & 3 & 1 & 2 & 3 & 1 & 2 & 3 \\
\hline$E(Y)$ & & & & & 1 & & & & \\
\hline$k_{q}$ & & & & & 3 & & & & \\
\hline$r_{f}$ & & & & & 0.00 & & & & \\
\hline$\sigma(Y)$ & & & & & 10.488 & & & & \\
\hline$K_{j}$ & & 2807.190 & & & 367.231 & & & 2006.917 & \\
\hline$\beta_{j}(k)$ & 3.0 & 3.0 & 3.0 & 3.8 & 3.8 & 3.8 & 4.6 & 4.6 & 4.6 \\
\hline$e_{j}(k)$ & 0.15 & 0.15 & 0.15 & 0.15 & 0.15 & 0.15 & 0.15 & 0.15 & 0.15 \\
\hline$\pi_{j}(k)$ & 1.10 & 1.10 & 1.10 & 1.117 & 1.117 & 1.117 & 1.083 & 1.083 & 1.083 \\
\hline$N_{j}(k)$ & 4500 & 4500 & 4500 & 3200 & 3200 & 3200 & 2300 & 2300 & 2300 \\
\hline equil/prem & 1.544 & 1.544 & 1.544 & 1.511 & 1.511 & 1.511 & 1.471 & 1.471 & 1.471 \\
\hline
\end{tabular}

Table 2. Scenario 1.

\begin{tabular}{|c|c|c|c|c|c|c|c|c|c|}
\hline \multirow{2}{*}{$\begin{array}{l}\text { Player } \\
\text { Period }\end{array}$} & \multicolumn{3}{|c|}{ I } & \multicolumn{3}{|c|}{ II } & \multicolumn{3}{|c|}{ III } \\
\hline & 1 & 2 & 3 & 1 & 2 & 3 & 1 & 2 & 3 \\
\hline$E(Y)$ & & & & & 1 & & & & \\
\hline$k_{q}$ & & & & & 3 & & & & \\
\hline$r_{f}$ & & & & & 0.05 & & & & \\
\hline$\sigma(Y)$ & & & & & 10.488 & & & & \\
\hline$K_{j}$ & & 2807.190 & & & 2367.231 & & & 2006.91 & \\
\hline$\beta_{j}(k)$ & 3.5 & 3.5 & 3.5 & 3.8 & 3.8 & 3.8 & 4.6 & 4.6 & 4.6 \\
\hline$e_{j}(k)$ & 0.15 & 0.15 & 0.15 & 0.15 & 0.15 & 0.15 & 0.15 & 0.15 & 0.15 \\
\hline$\pi_{j}(k)$ & 1.10 & 1.10 & 1.10 & 1.117 & 1.117 & 1.117 & 1.083 & 1.083 & 1.083 \\
\hline$N_{j}(k)$ & 4500 & 4500 & 4500 & 3200 & 3200 & 3200 & 2300 & 2300 & 2300 \\
\hline equil/prem & 1.494 & 1.494 & 1.494 & 1.488 & 1.488 & 1.488 & 1.449 & 1.449 & 1.449 \\
\hline
\end{tabular}

Finally, we assume that $\gamma_{i j}(k)=1, j=1, \cdots, 4 ; k=1,2,3$. Let the economic factor be -3 (which means that the economy is deteriorated) in Period 1, 0 in Period 2 and 3 (which means that the economy is raised) in Period 3. If the economic factor is equal to -3 , then the number of uninsured people (which corresponds to inactive state) increases up to $10000-N_{j}(1) \times 3=8701$. If economic factor is equal to 3 , then the number of uninsured people (which corresponds to inactive state) decreases down to $10000-N_{j}(3) \times 3=163$. The results are presented in Table 5. 
Table 3. Scenario 2.

\begin{tabular}{|c|c|c|c|c|c|c|c|c|c|}
\hline Player & \multicolumn{3}{|c|}{ I } & \multicolumn{3}{|c|}{ II } & \multicolumn{3}{|c|}{ III } \\
\hline Period & 1 & 2 & 3 & 1 & 2 & 3 & 1 & 2 & 3 \\
\hline$E(Y)$ & & & & & 1 & & & & \\
\hline$k_{q}$ & & & & & 3 & & & & \\
\hline$r_{f}$ & & & & & 0.05 & & & & \\
\hline$\sigma(Y)$ & & & & & 10.488 & & & & \\
\hline$K_{j}$ & & 2807.190 & & & 367.231 & & & 2006.917 & \\
\hline$\beta_{j}(k)$ & 3.0 & 3.5 & 3.0 & 3.8 & 3.9 & 3.8 & 4.6 & 5.6 & 4.6 \\
\hline$e_{j}(k)$ & 0.15 & 0.15 & 0.15 & 0.15 & 0.15 & 0.15 & 0.15 & 0.15 & 0.15 \\
\hline$\pi_{j}(k)$ & 1.10 & 1.10 & 1.10 & 1.117 & 1.117 & 1.117 & 1.083 & 1.083 & 1.083 \\
\hline$N_{j}(k)$ & 4500 & 4500 & 4500 & 3200 & 3200 & 3200 & 2300 & 2300 & 2300 \\
\hline equil/prem & 1.544 & 1.472 & 1.544 & 1.511 & 1.463 & 1.510 & 1.471 & 1.406 & 1.471 \\
\hline
\end{tabular}

Table 4. Scenario 3.

\begin{tabular}{|c|c|c|c|c|c|c|c|c|c|}
\hline \multirow{2}{*}{$\begin{array}{l}\text { Player } \\
\text { Period }\end{array}$} & \multicolumn{3}{|c|}{ I } & \multicolumn{3}{|c|}{ II } & \multicolumn{3}{|c|}{ III } \\
\hline & 1 & 2 & 3 & 1 & 2 & 3 & 1 & 2 & 3 \\
\hline$E(Y)$ & & & & & 1 & & & & \\
\hline$k_{q}$ & & & & & 3 & & & & \\
\hline$r_{f}$ & & & & & 0.05 & & & & \\
\hline$\sigma(Y)$ & & & & & 10.488 & & & & \\
\hline$K_{j}$ & & 2807.190 & & & 2367.231 & & & 2006.917 & \\
\hline$\beta_{j}(k)$ & 3.0 & 3.0 & 3.0 & 3.8 & 3.8 & 3.8 & 4.6 & 4.6 & 4.6 \\
\hline$e_{j}(k)$ & 0.15 & 0.15 & 0.15 & 0.15 & 0.15 & 0.15 & 0.15 & 0.15 & 0.15 \\
\hline$\pi_{j}(k)$ & 1.15 & 1.10 & 1.10 & 1.117 & 1.100 & 1.117 & 1.083 & 1.083 & 1.183 \\
\hline$N_{j}(k)$ & 4500 & 4500 & 4500 & 3200 & 3200 & 3200 & 2300 & 2300 & 2300 \\
\hline equil/prem & 1.580 & 1.538 & 1.579 & 1.527 & 1.499 & 1.544 & 1.487 & 1.466 & 1.542 \\
\hline
\end{tabular}

Table 5. Scenario 4.

\begin{tabular}{cccccccccc}
\hline Player & & I & & & II & & & III & \\
\hline Period & 1 & 2 & 3 & 1 & 2 & 3 & 1 & 2 & 3 \\
\hline$E(Y)$ & & & & & 1 & & & & \\
$k_{q}$ & & & & & 6.0 & & & & \\
$r_{f}$ & & & & & 0.05 & & & & \\
$\sigma(Y)$ & & & & & 10.488 & & & & \\
$K_{j}$ & & 2807.190 & & & 2367.231 & & & 2006.917 & \\
$\beta_{j}(k)$ & 3.0 & 3.0 & 3.0 & 3.8 & 3.8 & 3.8 & 4.6 & 4.6 & 4.6 \\
$e_{j}(k)$ & 0.15 & 0.15 & 0.15 & 0.15 & 0.15 & 0.15 & 0.15 & 0.15 & 0.15 \\
$\pi_{j}(k)$ & 1.10 & 1.10 & 1.10 & 1.117 & 1.117 & 1.117 & 1.083 & 1.083 & 1.083 \\
$N_{j}(k)$ & 433 & 2500 & 3279 & 433 & 2500 & 3279 & 433 & 2500 & 3279 \\
equil/prem & 1.676 & 1.633 & 1.676 & 1.619 & 1.590 & 1.638 & 1.685 & 1.660 & 1.741 \\
\hline & & & & & & & & &
\end{tabular}




\section{Conclusion}

In this paper, we aim to investigate an extension of the one-period model in non-life insurance markets (cf. [1]) by introducing a transition probability matrix depending on some economic factors. In the future, we concentrate on alternative ways of the extension including generalized Nash equilibrium (see, for instance [11] and [12]) formulations. Moreover, it would be interesting to investigate in more detail about economic factors that influence in our model.

\section{Acknowledgements}

The research funding was provided by the "L2766-MON: Higher Education Reform" project financed by the Asian Development Bank and executed by the Ministry of Education, Culture, Science and Sports of Mongolia.

\section{Conflicts of Interest}

The authors declare no conflicts of interest regarding the publication of this paper.

\section{References}

[1] Dutang, C., Albrecher, H. and Loisel, S. (2013) Competition among Non-Life Insurers under Solvency Constraints: A Game-Theoretic Approach. The European Journal of Operational Research, 231, 702-711. https://doi.org/10.1016/j.ejor.2013.06.029

[2] Albrecher, H. and Dalit, D-A. (2017) On Effects of Asymmetric Information on Non-Life Insurance Prices under Competition. International Journal of Data Analysis Techniques and Strategies, 9, 287-299. https://doi.org/10.1504/IJDATS.2017.088355

[3] Boonen, T.J., Pantelous, A.A. and Wu, R. (2018) Non-Cooperative Dynamic Games for General Insurance Markets. Insurance: Mathematics and Economics, 78, 123-135. https://doi.org/10.1016/j.insmatheco.2017.12.001

[4] Wu, R. and Pantelous, A.A. (2017) Potential Games with Aggregation in Non-Cooperative General Insurance Markets. ASTIN Bulletin, 47, 269-302. https://doi.org/10.1017/asb.2016.31

[5] Rees, R., Gravelle, H. and Wambach, A. (1999) Regulation of Insurance Markets. The Geneva Paper on Risk and Insurance Theory, 24, 55-68. https://doi.org/10.1023/A:1008733315931

[6] Durrett, R. (2012) Essentials of Stochastic Processes. Springer Texts in Statistics, Springer. https://doi.org/10.1007/978-1-4614-3615-7

[7] McFadden, D. (1981) Econometric Models of Probabilistic Choice, Structural Analysis of Discrete Data with Econometric Applications. The MIT Press, 198-272.

[8] Rosen, J.B. (1965) Existence and Uniqueness of Equilibrium Points for Concave N-Person Games. Econometrica, 33, 520-534.

[9] Facchinei, F. and Pang, J.S. (2003) Finite-Dimensional Variational Inequalities and Complementarity Problems. Computational Science \& Engineering, 2.

[10] Solodov, M.V. and Svaiter, B.F. (1999) A New Projection Method for Variational Inequality Problems. SIAM Journal on Control and Optimization, 37, 765-776. https://doi.org/10.1137/S0363012997317475 
[11] Altangerel, L. and Battur, G. (2012) Perturbation Approach to Generalized Nash Equilibrium Problems. Optimization Letters, 6, 1379-1391.

https://doi.org/10.1007/s11590-012-0510-8

[12] Facchinei, F. and Kanzow, C. (2010) Generalized Nash Equilibrium Problems. Annals of Operations Research, 175, 177-211.

https://doi.org/10.1007/s10479-009-0653-x 